
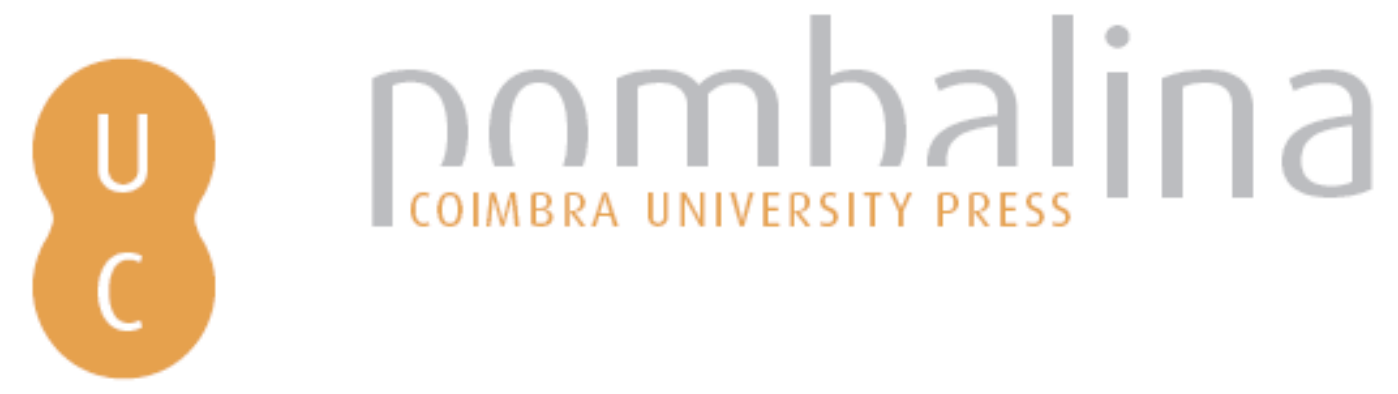

\title{
Quimioterapia combinada no tratamento do cancro: princípios e estratégias nanotecnológicas de entrega de fármacos
}

\author{
Autor(es): $\quad$ Pinto, Ana Catarina; Moreira, João Nuno; Simões, Sérgio \\ Publicado por: Imprensa da Universidade de Coimbra \\ URL \\ persistente: URI:http://hdl.handle.net/10316.2/36928 \\ DOI: $\quad$ DOI:http://dx.doi.org/10.14195/978-989-26-0881-5_17 \\ Accessed : $\quad$ 26-Apr-2023 15:18:34
}

A navegação consulta e descarregamento dos títulos inseridos nas Bibliotecas Digitais UC Digitalis, UC Pombalina e UC Impactum, pressupõem a aceitação plena e sem reservas dos Termos e Condições de Uso destas Bibliotecas Digitais, disponíveis em https://digitalis.uc.pt/pt-pt/termos.

Conforme exposto nos referidos Termos e Condições de Uso, o descarregamento de títulos de acesso restrito requer uma licença válida de autorização devendo o utilizador aceder ao(s) documento(s) a partir de um endereço de IP da instituição detentora da supramencionada licença.

Ao utilizador é apenas permitido o descarregamento para uso pessoal, pelo que o emprego do(s) título(s) descarregado(s) para outro fim, designadamente comercial, carece de autorização do respetivo autor ou editor da obra.

Na medida em que todas as obras da UC Digitalis se encontram protegidas pelo Código do Direito de Autor e Direitos Conexos e demais legislação aplicável, toda a cópia, parcial ou total, deste documento, nos casos em que é legalmente admitida, deverá conter ou fazer-se acompanhar por este aviso.

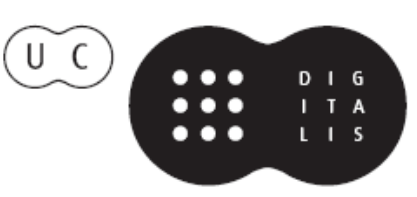


IOMATERIAIS APLICADOS AO DESENVOLVIMENTO DE SISTEMAS TERAPÊUTICOS AVANÇADOS

IOMATERIALES APLICADOS AL DISEÑO DE SISTEMAS TERAPÉUTICOS AVANZADOS

Hermínio C. de Sousa Mara E. M. Braga Alejandro Sosnik (editores) 


\title{
CAPÍTULO 17. QUIMIOTERAPIA COMBINADA NO TRATAMENTO DO CANCRO: PRINCÍPIOS E ESTRATÉGIAS NANOTECNOLÓGICAS DE ENTREGA D E F ÁRMACOS
}

\begin{abstract}
Ana Catarina Pinto ${ }^{1}$, João Nuno Moreira ${ }_{2}, 3$, Sérgio Simões ${ }^{1}{ }_{2}, 3$
${ }^{1}$ Bluepharma, Indústria Farmacêutica S.A., São Martinho do Bispo, Coimbra, Portugal ${ }_{2}$ Laboratório de Tecnologia Farmacêutica, Faculdade de Farmácia, Universidade de Coimbra, Coimbra, Portugal

3 Centro de Neurociências e Biologia Celular, Coimbra, Portugal
\end{abstract}

\section{Resumo:}

A quimioterapia combinada tem sido o tratamento padrão do cancro uma vez que pode permitir aumentar a resposta antitumoral assim como reduzir a resistência a fármacos. Uma combinação de fármacos pode resultar em efeitos sinergísticos, aditivos ou antagonistas dependendo do rácio de concentração. Vários métodos para avaliação quantitativa dos efeitos combinados de fármacos em linhas celulares têm sido usados e serão comparativamente revistos. A aplicação de sistemas nanotecnológicos de libertação controlada de fármacos, como os lipossomas, podem melhorar o índice terapêutico de alguns fármacos anticancerígenos ao aumentar a actividade antitumoral e/ou ao reduzir o seu perfil de toxicidade. A importância de desenvolver sistemas nanotecnológicos no sentido de modular as propriedades de fármacos anticancerígenos será discutida. A translação clínica de rácios de fármacos, previamente seleccionados in vitro, é complexa devido à farmacocinética e biodistribuição independentes revelada 
pelos fármacos individuais após administração intravenosa da sua combinação. A farmacocinética dissimilar resulta numa exposição das células tumorais a concentrações de fármaco inferiores à concentração mínima terapêutica ou a rácios antagonistas, com concomitante perda de actividade terapêutica. A aplicação de lipossomas como veículos de combinações de fármacos anticancerígenos tem sido descrita na literatura apenas recentemente. Os lipossomas têm a capacidade de sincronizar a farmacocinética e a biodistribuição de fármacos em combinação e entregá-los ao tecido tumoral num rácio fármaco-fármaco específico. A importância de manter um rácio óptimo de combinação de fármacos in vivo mediante encapsulação em lipossomas e a abordagem de "dosagem raciométrica" proposta por Lawrence Mayer e colegas será discutida.

Palavras-Chave: Quimioterapia combinada; método de análise do efeito médio; sistemas de libertação controlada de fármacos; lipossomas, rácio fármaco:fármaco

\begin{abstract}
:
Combination chemotherapy has been the standard of cancer treatment since it is a rationale strategy to increase efficacy and tolerability and to decrease resistance. A drug combination can result in synergistic, antagonistic or additive interaction effects at different concentration ratios. Several methods for the quantitative evaluation of drug-combined effects in cell culture systems have been used and will be comparatively reviewed. The application of nanotechnology-based drug delivery systems, such as liposomes, may improve overall therapeutic index of anticancer drugs by increasing their antitumor activity and/or by reducing their toxicity profile. The importance of developing drug delivery systems to modulate anticancer drug properties will be discussed. The translation of specific drug ratios, previously selected in vitro, to the clinical setting is complex due to the independent pharmacokinetics and biodistribution of individual drugs intravenously administered as free drug cocktail. The referred
\end{abstract}


uncoordinated pharmacokinetics results in exposure of tumor cells to drug concentrations below therapeutic threshold level or to antagonistic drug ratios with concomitant loss of therapeutic activity. The application of liposomes as carriers for anticancer drug combinations has been described in literature only in the last few years. Liposomes can synchronize pharmacokinetics and biodistribution of drug combinations and deliver them to tumor tissue at a specific drug ratio. The importance of maintaining an optimal drug combination ratio in vivo through drug encapsulation in liposomes and the "ratiometric dosing" approach proposed by Lawrence Mayer and colleagues will be discussed.

Keywords: Combination chemotherapy; median effect analysis; drug delivery systems; liposomes; drug-drug ratio. 


\subsection{Introdução}

O cancro é um sério problema de saúde pública, sendo a segunda causa de morte, apenas ultrapassado pelas doenças cardiovasculares [1]. O cancro é considerado o resultado de alterações quantitativas e estruturais em moléculas que controlam diferentes aspectos do ciclo de vida das células [2]. As alterações genéticas representam provavelmente o principal mecanismo molecular responsável pelo aparecimento, desenvolvimento e progressão do cancro [3]. Nos últimos anos têm sido realizados esforços significativos no sentido de identificar as modificações genéticas (hereditárias ou exógenas) mais comuns e os genes subjacentes responsáveis. Um artigo de revisão recente [2] classificou as 6 alterações na fisiologia celular características de uma doença oncológica: alterações na sinalização proliferativa, alterações nos mecanismos supressores de crescimento, ativação da invasão e de metástases, permissão de replicação imortalizada, indução de angiogénese, resistência à morte celular. Os agentes quimioterapêuticos utilizados na prática clínica atual têm revelado um impacto significativo na redução da mortalidade/morbilidade e no aumento da qualidade de vida dos pacientes [4].

Apesar dos avanços consideráveis no diagnóstico precoce e nos protocolos clínicos para tratamento do cancro, o desenvolvimento de fármacos que combinam eficácia, segurança e conveniência para o paciente são ainda um grande desafio para os investigadores [5].

Alguns fármacos anticancerígenos apresentam um índice terapêutico reduzido, desenvolvem resistência a múltiplos fármacos e revelam uma biodistribuição não específica após administração intravenosa, conduzindo a efeitos adversos inaceitáveis em tecidos saudáveis, nomeadamente na medula óssea e no trato gastrointestinal. Estas limitações das estratégias quimioterapêuticas convencionais resultam frequentemente em dosagens sub-terapêuticas, atraso no tratamento e reduzida aceitação do paciente [6]. 


\subsection{Introdução quimioterapia combinada}

\subsubsection{Princípios e vantagens}

A quimioterapia combinada tem sido a estratégia padrão no tratamento de cancro uma vez que se trata de uma abordagem terapêutica que pode permitir aumentar a resposta antitumoral assim como reduzir a resistência a fármacos. No presente, observa-se um interesse crescente em combinar fármacos anticancerígenos ambicionando um aumento de eficácia e/ou uma redução ou minimização da toxicidade sistémica mediante entrega de uma dose menor de fármacos [6-8].

Os princípios da quimioterapia combinada têm permanecido inalterados ao longo das últimas décadas: a) os fármacos devem apresentar toxicidades não sobreponíveis para que cada fármaco possa ser usado numa dose próxima da dose máxima tolerada; b) os fármacos devem ter mecanismos de ação distintos e que minimizem a ocorrência de resistência; c) os fármacos devem demonstrar atividade anti-tumoral enquanto individuais; d) a administração deve ocorrer, preferencialmente, num estádio inicial da doença e em ciclos espaçados no tempo de forma a permitir a recuperação dos tecidos mais sensíveis à quimioterapia [6-9].

As vantagens apresentadas pela quimioterapia combinada incluem uma maior adesão do doente a terapêutica devido à redução no número de administrações, a possibilidade de ocorrência de aditividade ou sinergia dos efeitos anti-tumorais que permita a redução da dose de pelo menos um fármaco, com consequente redução dos efeitos adversos associados [7, 10, 11]. Dada a complexidade, heterogeneidade e resistência a tratamento da maioria dos cancros, uma combinação de fármacos racionalmente desenvolvida é necessária para alcançar um progresso significativo no tratamento desta doença. 


\subsubsection{Estudos pré-clínicos e clínicos de combinação de fármacos}

A maioria dos protocolos clínicos inclui uma combinação de fármacos escolhida de forma algo empírica, sem dados experimentais de suporte e baseados no estudo retrospetivo dos resultados obtidos em ensaios clínicos anteriores [8, 12]. Estes estudos, dispendiosos e morosos, investigam a sequência e a posologia de administração dos fármacos mas não têm em conta os efeitos interativos resultantes da combinação dos dois fármacos [8].

É muito difícil determinar se uma combinação de fármacos resulta em efeitos anti-tumorais sinergísticos, aditivos ou antagonistas. Normalmente, é sim possível determinar se um tratamento combinado promove um aumento estatisticamente significativo de um endpoint, como por exemplo a redução do tamanho de um tumor, a redução dos efeitos secundários ou o aumento da esperança de vida [6].

Os estudos pré-clínicos de interação de fármacos anticancerígenos permitem a preparação de um protocolo clínico de quimioterapia mais adequado e eficaz. O princípio de que "quanto maior a dose de fármacos melhor" é um pressuposto incorreto pois pode resultar numa maior toxicidade e/ou menor eficácia do tratamento uma vez que a maioria dos efeitos resultantes da interação entre os fármacos são dependentes das suas concentrações. Indiscutivelmente, existem fatores moleculares e farmacológicos que determinam a eficácia de uma combinação de fármacos anticancerígenos $[7,13]$. A preparação experimental de um estudo pré-clínico de combinação de fármacos anti-tumorais realizado com linhas celulares ou modelos animais tem de ter em conta diversos fatores, nomeadamente a concentração de cada fármaco, o tempo de exposição, a posologia e o método utilizado para quantificar a interação entre os fármacos [8].

\subsubsection{Estudos in vitro e in vivo com combinação de fármacos}

A avaliação dos efeitos anti-tumorais resultantes de uma quimioterapia combinada é normalmente realizada em cultura de linhas celulares. 
Estes estudos in vitro apresentam diversas vantagens: a) as condições experimentais são flexíveis e podem ser rigorosamente controladas; b) a quantificação de ácidos nucleicos e de proteína é fácil e c) a avaliação quantitativa da inibição de crescimento e/ou de morte celular é rigorosa [8]. No entanto, os estudos in vitro com linhas celulares têm uma utilidade limitada uma vez que as condições experimentais são artificiais e não refletem a heterogeneidade e complexidade clínicas de uma doença cancerígena [14]. Os modelos animais com tumor representam um sistema dinâmico, onde os fármacos sofrem absorção, distribuição, metabolismo e eliminação, conduzindo, portanto, a alterações na concentração plasmática dos fármacos [15]. Quando comparados com os estudos in vitro, a determinação de sinergismo ou antagonismo em estudos in vivo é mais difícil, morosa e dispendiosa. Neste sentido, geralmente a avaliação in vitro ocorre em primeiro lugar com diversas combinações de fármacos, enquanto nos estudos em animais são apenas selecionadas as combinações de fármacos que produziram os melhores efeitos anti-tumorais in vitro [10, 11].

\subsection{Efeitos de interação de fármacos na quimioterapia combinada}

\subsubsection{Definição e avaliação quantitativa in vitro}

Uma combinação de fármacos pode resultar em efeitos de interação sinergísticos, aditivos ou antagonistas dependendo do rácio de concentração dos fármacos. Sinergismo, aditividade e antagonismo são definidos como o resultado da interação de dois fármacos em que o efeito combinado é superior, igual ou inferior, respetivamente, à soma dos efeitos individuais $[11,15]$.

A ocorrência de sinergismo num ensaio in vitro com células demonstra uma forte dependência do rácio fármaco: fármaco, e essa dependência têm sérias implicações na utilização clínica uma vez que a atividade da combinação in vivo depende da manutenção do rácio terapêutico no local alvo, como por exemplo um tumor [6, 9, 13]. 
Vários métodos têm sido usados para realizar a avaliação quantitativa dos efeitos de interação resultantes da combinação de dois fármacos [8, 15]. A revisão destes métodos não é um objectivo do presente capítulo mas uma breve descrição dos seus princípios e limitações é apresentada na Tabela 17.1. No entanto, o método de análise do efeito médio será descrito em maior detalhe na próxima seção uma vez que é o método mais utilizado e revisto na literatura.

Tabela 17.1. Métodos para avaliação quantitativa dos efeitos de interação resultantes da combinação de dois fármacos.

\begin{tabular}{|c|c|c|c|}
\hline Método & Autor & Princípio & Limitações \\
\hline $\begin{array}{l}\text { Produto } \\
\text { fracional }\end{array}$ & Webb (1963) & $\begin{array}{l}\text { A soma dos efeitos de dois } \\
\text { inibidores é expressa pelo pro- } \\
\text { duto das atividades fracionais }\end{array}$ & $\begin{array}{l}\text { O método não tem em conta a pos- } \\
\text { sível sigmoicidade da curva dose- } \\
\text {-resposta }(\mathrm{m}>1 \text { ou } \mathrm{m}<1) \text { e não é } \\
\text { aplicável a fármacos com o mesmo } \\
\text { mecanismo de ação ou semelhante }\end{array}$ \\
\hline $\begin{array}{l}\text { Isobo- } \\
\text { lograma } \\
\text { clássico }\end{array}$ & Loewe (1957) & $\begin{array}{l}\text { Linhas no gráfico unem doses } \\
\text { de fármaco que exercem o } \\
\text { mesmo efeito }\end{array}$ & \multirow{2}{*}{$\begin{array}{l}\text { O método requer um número } \\
\text { elevado de dados, não possui um } \\
\text { software computacional aplicável, } \\
\text { a abordagem estatística é incom- } \\
\text { pleta. Não é aplicável a combina- } \\
\text { ções com mais de } 2 \text { fármacos }\end{array}$} \\
\hline $\begin{array}{l}\text { Isobolo- } \\
\text { grama mo- } \\
\text { dificado }\end{array}$ & $\begin{array}{l}\text { Stell and } \\
\text { Peckham } \\
(1979)\end{array}$ & $\begin{array}{l}\text { Existência de um "envelope de } \\
\text { aditividade": região delimitada } \\
\text { por limites de confiança, em } \\
\text { que os fármacos não intera- } \\
\text { gem significativamente }\end{array}$ & \\
\hline $\begin{array}{l}\text { Análise } \\
\text { do efeito } \\
\text { médio }\end{array}$ & $\begin{array}{l}\text { Chou and Ta- } \\
\text { lalay (1984) }\end{array}$ & $\begin{array}{l}\text { Sistema de cinética enzimática: } \\
\text { lei de ação das massas, equa- } \\
\text { ções de Michaelis-Menten. }\end{array}$ & $\begin{array}{l}\text { Método mais usado e revisto lite- } \\
\text { ratura mas não é aplicável quan- } \\
\text { do as curvas dose-resposta não } \\
\text { são sigmoidal dada a dificuldade } \\
\text { de aplicar uma regressão linear } \\
\text { posteriormente }\end{array}$ \\
\hline
\end{tabular}

Adaptação de diferentes referências bibliográficas [8, 11, 15, 16]

\subsubsection{O método de Análise do efeito médio}

O método mais utilizado e referido na literatura para análise quantitativa dos efeitos gerados por uma combinação de fármacos é o método da Análise do Efeito Médio proposto por Chou e Talalay [10, 11, 16, 17]. As equações fundamentais deste método foram derivadas dos modelos de cinética enzimática, previamente estabelecidos para as interações enzima-substrato e, posteriormente, aplicáveis a combinações de fármacos [18]. 
Independente da forma da curva dose-resposta (Figura 17.1) ou do mecanismo de ação, a equação do efeito médio correlaciona, para cada fármaco, a dose e o seu efeito correspondente (ex. inibição do crescimento celular) e é descrita pela fórmula:

$$
\mathrm{f}_{\mathrm{a}} / \mathrm{f} \underline{\mathrm{u}}=\left(\mathrm{D} / \mathrm{D}_{\mathrm{m}}\right)^{\mathrm{m}}
$$

Em que " $\mathrm{f}_{\mathrm{a}}$ " $\mathrm{e}$ " $\mathrm{f}_{\mathrm{u}}$ " são as frações celulares afetadas e não afetadas, respetivamente, por uma dose " $\mathrm{D}$ ". " $\mathrm{D}_{\mathrm{m}}$ ” é a dose que induz o efeito médio e " $m$ " é o coeficiente que traduz a forma da curva dose-efeito $(m=1, m>$ 1 e $\mathrm{m}<1$, indicam curvas hiperbólica, sigmoidal e sigmoidal negativa, respectivamente). Os parâmetros " $\mathrm{m}$ " e " $\mathrm{D}_{\mathrm{m}}$ " são facilmente determinados a partir recta do efeito médio uma vez que correspondem ao declive e interseção na origem [11, 19]. Uma vez determinados os parâmetros "m" e $\mathrm{D}_{\mathrm{m}}$ ", a relação dose-efeito é completamente descrita, ou seja, para uma determinada dose do fármaco 1 e fármaco 2 combinados é possível calcular o efeito correspondente $\left(f_{a}\right)$ e vice-versa $[10,17]$. A aplicação da Equação 17.1 permite a linearização da das curvas hiperbólicas $(\mathrm{m}=1)$ ou sigmoidais $(m \neq 1)$. A aplicação do logaritmo à Equação $17.1(x=\log (\mathrm{D})$ vs. $\mathrm{y}=$ $\log \left(\mathrm{f}_{\mathrm{a}} / \mathrm{f}_{\mathrm{u}}\right)$ ) origina o gráfico do efeito médio (median effect plot) $[10,11](1)$.

\section{A. Curvas Dose-Efeito}

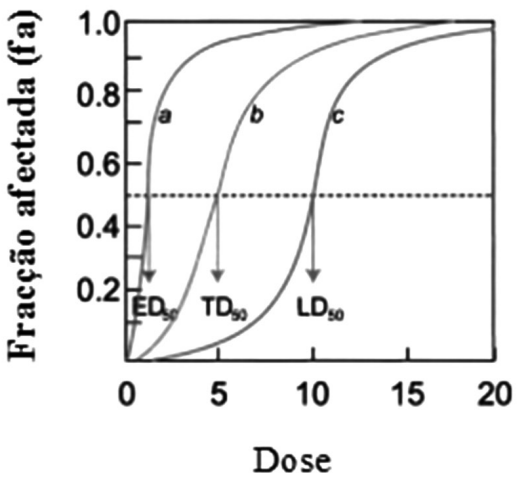

B. Plots do Efeito Médio

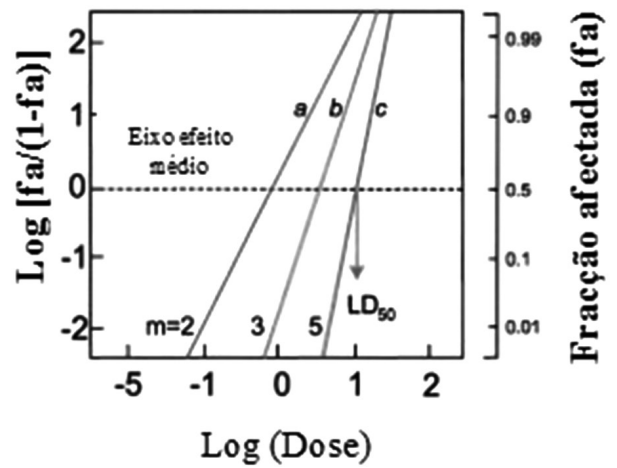

Figura 17.1. Representações gráficas originadas pelo método de análise do efeito médio. Três curvas sigmoidais $(\mathrm{a}, \mathrm{b}, \mathrm{c})$ (gráfico à esquerda) são transformadas na respetiva forma linear originando as rectas do efeito médio, em que $y=\log \left(\mathrm{f}_{\mathrm{a}} /\right.$ $\mathrm{f}_{\mathrm{u}}$ ) vs. $\mathrm{x}=\log (\mathrm{D})$. Adaptado da referência bibliográfica [11]. 
A conformidade dos dados com o Principio do Efeito Médio é facilmente traduzida pelo coeficiente de correlação linear da recta (r) que quanto mais perto do valor 1 , traduz maior conformidade [10, 11, 17]. O gráfico do efeito médio (median effect plot) apresenta rectas paralelas se os fármacos apresentarem mecanismos de ação iguais ou semelhantes e, assim, os efeitos resultantes da combinação denominam-se mutuamente exclusivos. Se as rectas de cada fármaco forem paralelas mas o gráfico correspondente à combinação não é uma recta mas sim uma linha côncava que intersecta a recta do gráfico mais potente significa que os fármacos atuam independentemente e os seus efeitos são considerados mutuamente não exclusivos [16].

O Índice de Combinação (do Inglês combination index (CI)) traduz quantitativamente o tipo de interação entre os fármacos e é definido pela seguinte equação:

$$
\mathrm{CI}=\frac{(D)_{1}}{\left(D_{x}\right)_{1}}+\frac{(D)_{2}}{\left(D_{x}\right)_{2}}+\alpha \frac{(D)_{1}(D)_{2}}{\left(D_{x}\right)_{1}\left(D_{x}\right)_{2}}
$$

Onde $\alpha=0$ e $\alpha=1$ para fármacos com mecanismos de ação mutuamente exclusivos e não exclusivos, respectivamente $[10,11,16,17]$.

Os denominadores $\left(D_{\mathrm{x}}\right)_{1}$ and $\left(\mathrm{D}_{\mathrm{x}}\right)_{2}$ são as doses de cada fármaco necessárias para originar um nível de efeito $\left(f_{a}\right)$. Os numeradores (D) $)_{1}$ and (D) 2 são as doses de cada fármaco que quando combinados originam o mesmo nível de efeito $\left(\mathrm{f}_{\mathrm{a}}\right)$. No caso de combinações de três fármacos, um terceiro termo $(D)_{3} /\left(D_{x}\right)_{3}$ é adicionado à equação 17.2.

O gráfico que traduz o Índice de combinação $(C I)$ em função do efeito $\left(f_{a}\right)$ está exemplificado na Figura 17.2. Valores de CI inferiores, iguais ou superiores a 1 traduzem sinergismo, aditividade e antagonismo, respectivamente.

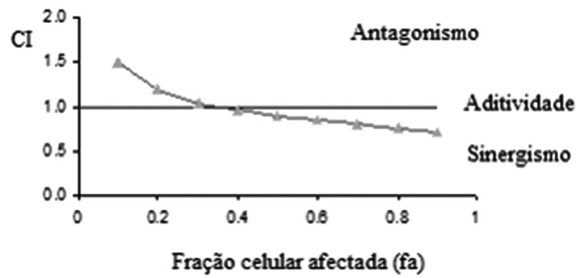

Figura 17.2. Representações gráfica exemplificativa de uma curva CI vs $f_{a}$. Valores de $\mathrm{CI}<1,=1,>1$, indicam sinergismo, aditividade e antagonismo, respectivamente. 
Se o tipo de mecanismo de ação dos fármacos testados não é conhecido ou não é claro, os autores do método sugerem que o valor do Índice de Combinação seja calculado assumindo que os efeitos resultantes da combinação são mutualmente exclusivos $(\alpha=0)$ e mutualmente não exclusivos $(\alpha=1)$. A segunda determinação é mais conservadora uma vez que a adição do terceiro resulta em valores de CI mais elevados que a primeira abordagem [11, 16, 17].

Apesar de o índice de Combinação poder ser calculado para qualquer nível de efeito $\left(f_{a}\right)$, a determinação mais rigorosa ocorre para $f_{a}=0.5$ uma vez que o plot do efeito médio apresenta-se pouco fidedigno nos extremos uma vez que se trata da representação linear de uma função não linear [20].

Em suma, a Análise do Efeito Médio é um método quantitativo simples de realizar e que tem em conta não só a dose de cada fármaco (D) mas também a forma (hiperbólica ou sigmoidal) da curva dose-resposta $[17,19]$. Adicionalmente, este método permite avaliar os efeitos de interação para diferentes rácios de fármaco e três fármacos podem ser combinados e avaliados [16, 19]. É recomendado que a experiência in vitro seja realizada com o rácio fármaco 1: fármaco 2 equipotente (ex. $\left.\left(\mathrm{IC}_{50}\right)_{1} /\left(\mathrm{IC}_{50}\right)_{2}\right)$ para que a contribuição de efeito de cada fármaco na mistura seja semelhante $[10,11,16]$. Quando se avaliam combinações de fármacos anticancerígenos, a gama de doses testada deve ser suficientemente abrangente para permitir a extrapolação de resultados para níveis de atividade antitumoral elevados $\left(f_{a} \geq 0.5\right)$ uma vez que valores inferiores não têm significado clínico [9, 10, 15].

O Índice de redução da dose (do Inglês, Dose Reduction Index (DRI)) é um valor que traduz quantas vezes a dose de um fármaco pode ser reduzida por ele se encontrar em combinação para um dado nível de efeito anti-tumoral, comparativamente com o fármaco individual [11, 13]. O DRI é um parâmetro muito importante na prática clínica uma vez que o valor superior a significa uma redução da dose de 1 ou dos 2 fármacos por se encontrarem combinados, com consequente redução da toxicidade sistémica nos tecidos saudáveis, mantendo porém a mesma eficácia terapêutica $[10,11,16,21]$. 


\subsection{Nanopartículas como sistema de entrega de fármacos}

Perante as dificuldades e custos crescentes associados ao desenvolvimento de novas entidades químicas com atividade terapêutica, a estratégia de algumas indústrias farmacêuticas adotada baseado na optimização dos seus fármacos já comercializados, principalmente aqueles com menor índice terapêutico, como por exemplo os fármacos anticancerígenos.

Em particular, a aplicação de estratégias nanotecnológicas como sistema de entrega de fármacos na quimioterapia do cancro tem se revelando uma área de investigação muito prometedora e constitui um esforço significativo para melhorar a especificidade e eficácia dos fármacos anticancerígenos.

\subsubsection{Os diferentes tipos de nanopartículas}

Vários fármacos possuem propriedades físicas e biológicas que limitam a sua aplicação clínica, nomeadamente baixa solubilidade em água, rápido metabolismo, instabilidade sob condições fisiológicas, farmacocinética desfavorável e distribuição não específica para tecidos saudáveis [22]. No caso particular dos fármacos anticancerígenos estas propriedades originam entrega de concentrações sub-terapêuticas aos tecidos tumorais e/ou efeitos secundários inaceitáveis [23-24]. Nesse sentido, é crucial o desenvolvimento de sistemas nanotecnológicos (de base lipídica ou polimérica) de entrega de fármacos (lipossomas, micelas, nanoparticulas polimérica, dendrímeros) para promover e controlar a entrega de fármaco(s) aos tecidos tumorais [25-27]. Nanopartículas com aplicação médica diferem em termos de estrutura, tamanho e composição, o que se traduz em diferentes características, nomeadamente capacidade de carga de fármaco, estabilidade física e especificidade de entrega de fármaco [28]. Não é objetivo do presente capítulo rever o tipo, a aplicação e as propriedades das diferentes nanotransportadores mas este tema encontra-se amplamente descrito e discutido em várias referências bibliográficas [25-31]. O presente capítulo irá apresentar uma revisão global dos lipossomas como sistema de entrega de fármacos, individuais ou combinados. 


\subsubsection{Lipossomas}

\subsubsection{Definição geral e principais propriedades}

Os lipossomas, descritos pela primeira vez por Bangham et al. [32] são vesículas lipídicas (Figura 17.3) com um elevado potencial para a entrega de fármacos dadas as suas características inerentes: biocompatibilidade, biodegradabilidade, simplicidade de desenvolvimento, produção em escala, toxicidade reduzida, fraca imunogenicidade e versatilidade na sua estrutura e nas suas características físico-químicas (tamanho, composição lipídica, carga, conjugação com ligando à superfície) [24, 33, 34]. Mediante estas propriedades tão específicas, os lipossomas têm a capacidade de controlar o comportamento in vivo (farmacocinética e perfil de biodistribuição) e/ ou modular a solubilidade dos fármacos e protegê-los da degradação precoce após administração intravenosa [33, 35, 36]. De uma maneira geral, quando um fármaco é encapsulado num transportador, com por exemplo o lipossoma (Figura 17.3), o volume de distribuição e a eliminação plasmática diminuem, enquanto o tempo de permanência no plasma e a área sob a curva concentração vs. tempo aumentam [37].

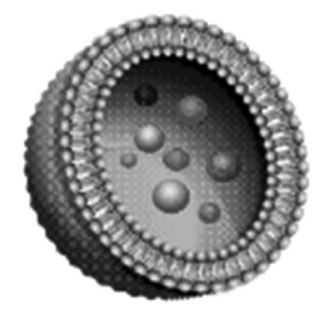

Figura 17.3. Representação esquemática geral de um lipossoma enquanto veículo transportador de moléculas de fármaco encapsuladas no seu compartimento aquoso central.

O desenvolvimento bem-sucedido de uma formulação lipossomal encapsulando um ou mais fármacos deve cumprir com três requisitos fundamentais: a) compreensão da fisiologia e biologia da doença a tratar; b) 
conhecimento profundo das propriedades físico-químicas do transportador e do fármaco(s) e c) determinação das alterações na farmacocinética e biodistribuição do fármaco induzidas pelo veículo lipossomal [22].

Os tipos de lipossomas, as diferentes composições lipídicas e métodos de preparação não serão alvo de revisão no presente capítulo mas essa informação poderá ser consultada em diversas referências bibliográficas publicadas pelos grupos de investigação da Dra. Allen [22, 38, 39] e do Dr. Lasic [40].

\subsubsection{Aplicações médicas}

Nas últimas décadas o desenvolvimento de formulações lipossomais de fármacos tem sofrido uma grande evolução, o que permitiu aumentar a sua gama de aplicações médicas: tratamento do cancro, de infeções, de doenças oftalmológicas, terapia génica, imagiologia de diagnóstico, vacina, terapia fotodinâmica, dermatologia, transportador de hemoglobina ou de enzimas [33, 35, 41-43]. O principal objetivo de uma formulação lipossomal destinada ao tratamento do cancro é melhorar o índice terapêutico do(s) fármaco(s) encapsulados mediante aumento da atividade antitumoral e/ou redução do perfil de toxicidade devido a uma entrega e acumulação preferencial no tumor comparativamente com os fármacos na forma livre após administração intravenosa [36, 44].

\subsubsection{Comportamento in vivo dos lipossomas}

Idealmente, uma formulação lipossomal de um fármaco deve possuir um diâmetro próximo de $100 \mathrm{~nm}$, um rácio fármaco: lípido elevado, uma boa retenção do(s) fármaco(s) durante a circulação sanguínea e um tempo de circulação longo (desde horas até dias) [45].

De uma forma geral, um tempo de circulação sanguínea reduzido e a libertação precoce do(s) fármaco(s) a partir do lipossoma convencional limita a sua aplicabilidade clínica. Os lipossomas convencionais 
são reconhecidos e ligados por proteínas (opsoninas) e pelo sistema complemento plasmático e, de seguida, são removidos da circulação sistémica pelas células do sistema reticulo-endotelial do fígado, baço e medula óssea [33, 46, 47]. As propriedades físico-químicas dos lipossomas (tamanho, carga, hidrofobicidade, fluidez da bicamada) influenciam a estabilidade física em termos de retenção do(s) fármaco(s) e o tipo de proteínas e lipoproteínas plasmáticas que se ligam [33, 46]. O uso de lípidos saturados na bicamada lipossomal (que possuam uma temperatura de transição de fase elevada) conjuntamente com colesterol, mas principalmente o acoplamento à superfície dos lipossomas de polímeros hidrofílicos (ex. polietilenoglicol - PEG) aumentam significativamente (de horas para dias) o tempo de circulação plasmática e a estabilidade das formulações lipossomais [33, 37, 39, 47]. Os lipossomas que apresentam ligando hidrofílicos à superfície revelam menor adsorção e ligação de opsoninas e são denominados lipossomas estabilizados estereamente (do inglês, sterically stabilized liposomes (SSL) or Stealth $\left.{ }^{\circledR}\right)[23,33,37]$.

\subsubsection{Comportamento in vivo dos lipossomas}

A maioria dos tumores sólidos possui características fisiológicas únicas que se encontram ausentes em tecidos saudáveis, nomeadamente angiogénese muito extensa e descontrolada, arquitetura vascular caótica, permeabilidade vascular aumentada e drenagem linfática limitada [48, 49]. Este fenómeno denominado efeito da permeabilidade e da retenção aumentados (do inglês, enhanced permeability and retention (EPR) effect) (Figura 17.4) [36, 49] tem sido amplamente descrito em diversos tumores experimentais e depende do tipo, volume e vascularização tumoral, assim como da permeabilidade dos vasos sanguíneos [48, 50]. Devido a este efeito os lipossomas estabilizados estereamente e com um diâmetro na gama dos 100-200 nm demonstram extravasação preferencial através da vasculatura tumoral muito permeável e acumulação passiva no espaço intersticial devido à drenagem linfática ineficaz (Figura 17.4). 


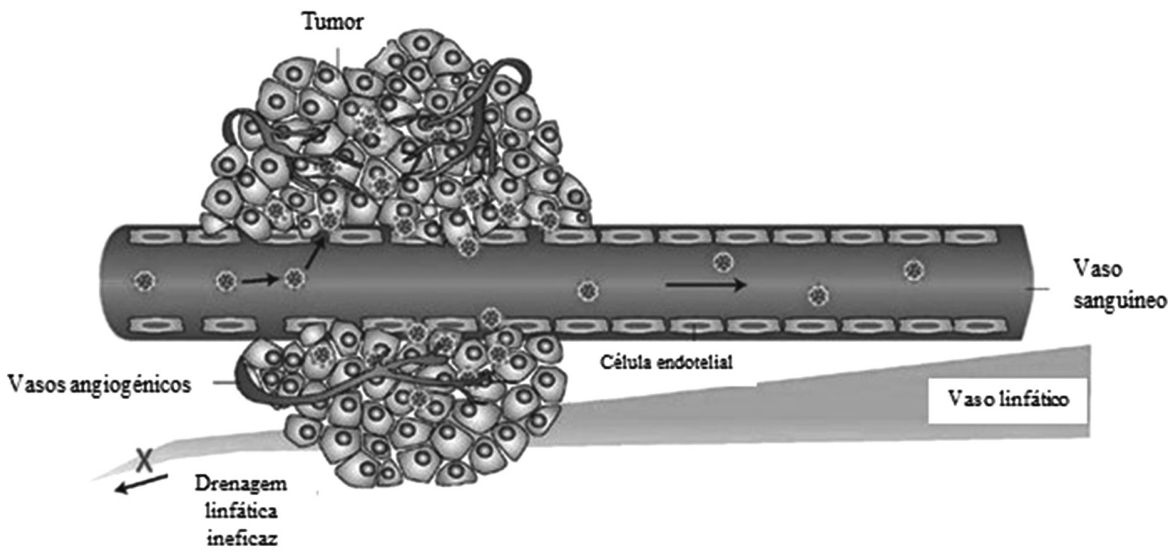

Figura 17.4. Efeito da permeabilidade e da retenção aumentados (do inglês, enhanced permeability and retention (EPR) effect). Os lipossomas são representativos de nanopartículas. O direcionamento para o tumor é conseguido de forma passiva devido à extravasação dos lipossomas da corrente sanguínea para o espaço intersticial tumoral devido à vasculatura muito permeável e à retenção e acumulação preferenciais devido a um sistema de drenagem linfática ineficaz. Adaptado da referência bibliográfica [26].

\subsubsection{Formulações lipossomais de fármacos individuais apro-} vadas ou em desenvolvimento clínico

O sucesso dos lipossomas enquanto sistema de transporte e entrega de fármaco(s) é refletido pelo número significativo de produtos aprovados para uso clínico pelas autoridades regulamentares (FDA - Food and drug Administration e EMA - European Medicines Agency) (Tabela 17.2) ou em desenvolvimento em ensaios clínicos (Tabela 17.3).

Exemplos de formulações lipossomais de um fármaco individual são o Doxil ${ }^{\circledR}$ e o Myocet ${ }^{\circledR}$ e que estão aprovados no tratamento de diversos cancros (Tabela 17.2). A encapsulação da doxorrubicina num lipossoma permitiu reduzir significativamente os efeitos tóxico associados a este fármaco, nomeadamente cardiomiopatia, efeitos hematológicos, alopecia e náuseas, sem alterar a sua atividade terapêutica $[37,51,52]$. 
Tabela 17.2. Formulações lipossomais de um fármaco individual aprovadas para aplicação clínica.

\begin{tabular}{|c|c|c|c|c|}
\hline $\begin{array}{c}\text { Nome } \\
\text { comercial }\end{array}$ & Substância ativa & Tipo de formulação & $\begin{array}{l}\text { Via de admi- } \\
\text { nistração }\end{array}$ & $\begin{array}{l}\text { Indicação } \\
\text { terapêutica }\end{array}$ \\
\hline Abelcet $^{\circledR}$ & Anfotericina B & Complexo lipídico & Intravenosa & $\begin{array}{l}\text { Infeção fúngica } \\
\text { sistémica }\end{array}$ \\
\hline Ambisome $^{\circledR}$ & Anfotericina B & Lipossoma & Intravenosa & $\begin{array}{l}\text { Infeção fúngica } \\
\text { sistémica }\end{array}$ \\
\hline Amphotec $^{\circledR}$ & Anfotericina B & Complexo lipídico & Intravenosa & $\begin{array}{l}\text { Infeção fúngica } \\
\text { sistémica }\end{array}$ \\
\hline DaunoXome ${ }^{\circledR}$ & Daunorrubicina & Lipossoma & Intravenosa & $\begin{array}{l}\text { Tumores hemato- } \\
\text { lógicos }\end{array}$ \\
\hline $\begin{array}{l}\text { Doxil }^{\circledR} \text { (USA) } \\
\text { Caelyx }^{\circledR}(\mathrm{EU})\end{array}$ & Doxorrubicina & $\begin{array}{l}\text { Lipossoma PEGui- } \\
\text { lado }\end{array}$ & Intravenosa & $\begin{array}{l}\text { Sarcoma de Kaposi } \\
\text { Cancro ovário e da } \\
\text { mama avançados } \\
\text { Mieloma múltiplo }\end{array}$ \\
\hline Lipo-dox & Doxorrubicina & $\begin{array}{l}\text { Lipossoma PEGui- } \\
\text { lado }\end{array}$ & Intravenosa & $\begin{array}{l}\text { Sarcoma de Kaposi } \\
\text { Cancro ovário e da } \\
\text { mama avançados }\end{array}$ \\
\hline Myocet $^{\circledR}$ & Doxorrubicina & Lipossoma & Intravenosa & $\begin{array}{l}\text { Cancro da mama } \\
\text { (combinação com } \\
\text { ciclofosfamida) }\end{array}$ \\
\hline Visudyne $^{\circledR}$ & Verteporfina & Lipossoma & Intravenosa & $\begin{array}{l}\text { Degeneração ma- } \\
\text { cular relacionada à } \\
\text { idade } \\
\text { Miopia patológica }\end{array}$ \\
\hline DepoCyt ${ }^{\circledR}$ & Citarabina & Lipossoma & Vertebral & $\begin{array}{l}\text { Meningite } \\
\text { linfomatosa } \\
\text { Meningite } \\
\text { neoplásica } \\
\end{array}$ \\
\hline DepoDur ${ }^{\circledR}$ & Sulfato de morfina & Lipossoma & Epidural & Controlo da dor \\
\hline Epaxal ${ }^{\circledR}$ & $\begin{array}{l}\text { Vírus Hepatite A } \\
\text { inativado (estirpe } \\
\text { RG-SB) }\end{array}$ & Lipossoma & Intramuscular & Hepatite A \\
\hline Inflexal V & $\begin{array}{l}\text { Hemaglutinina } \\
\text { inativada do vírus } \\
\text { influenza (estirpes } \\
\text { A e B) }\end{array}$ & Lipossoma & Intramuscular & $\begin{array}{l}\text { Infeção pelo vírus } \\
\text { Influenza }\end{array}$ \\
\hline Exparel $^{\circledR}$ & Bupivacaína & Lipossoma & i.v. & Controlo da dor \\
\hline
\end{tabular}

Informações recolhidas na referência bibliográfica [53] e nas páginas oficiais na internet da USA Food and Drug Administration (FDA) - http://www.fda.gov (2012) e da European Medicines Agency (EMA) - http://www.ema.europa.eu (2012). 
Tabela 17.3. Exemplos de formulações lipossomais de um fármaco individual em desenvolvimento clínico para tratamento do cancro.

\begin{tabular}{|c|c|c|c|c|}
\hline $\begin{array}{c}\text { Nome } \\
\text { comercial }^{\mathrm{a}}\end{array}$ & $\begin{array}{l}\text { Substância } \\
\text { ativa }\end{array}$ & $\begin{array}{l}\text { Via de admi- } \\
\text { nistração }\end{array}$ & $\begin{array}{l}\text { Indicação } \\
\text { terapêutica }\end{array}$ & Fase \\
\hline LEP-ETU & Paclitaxel & Intravenosa & $\begin{array}{l}\text { Cancros do ovário, mama e } \\
\text { pulmão }\end{array}$ & $\mathrm{I} / \mathrm{II}$ \\
\hline LEM-ETU & Mitoxantrona & Intravenosa & $\begin{array}{l}\text { Leucemia e cancros da mama, } \\
\text { estômago, fígado e ovário }\end{array}$ & I \\
\hline EndoTAG-1 & Paclitaxel & Intravenosa & $\begin{array}{l}\text { Cancros da mama e do pân- } \\
\text { creas }\end{array}$ & II \\
\hline Arikace & Amicacina & Aerossol & Infeção pulmonar & III \\
\hline Marqibo & Vincristina & Intravenosa & Melanoma metastático & III \\
\hline ThermoDox & Doxorrubicina & Intravenosa & $\begin{array}{l}\text { Cancro hepático não resse- } \\
\text { cável }\end{array}$ & III \\
\hline Atragen & Tretinoína & Intravenosa & $\begin{array}{l}\text { Leucemia aguda promielo- } \\
\text { cítica } \\
\text { Cancro da próstata refratário } \\
\text { a hormonas }\end{array}$ & II \\
\hline Nyotran & Nistatina & Intravenosa & Infeções fúngicas sistémicas & $\mathrm{I} / \mathrm{II}$ \\
\hline LE-SN38 & $\begin{array}{l}\text { Metabolito ativo do } \\
\text { irinotecano (SN-38) }\end{array}$ & Intravenosa & Cancro coloretal metastático & $\mathrm{I} / \mathrm{II}$ \\
\hline Aroplatin & $\begin{array}{l}\text { Análogo da cisplatina } \\
\text { (L-NDDP) }\end{array}$ & Intrapleural & Cancro coloretal metastático & II \\
\hline Liprostin & Prostaglandina E1 & Intravenosa & Doença vascular periférica & II/III \\
\hline SPI-077 & Cisplatina & Intravenosa & $\begin{array}{l}\text { Cancros da cabeça / pescoço } \\
\text { e do pulmão }\end{array}$ & $\mathrm{I} / \mathrm{II}$ \\
\hline Lipoplatin & Cisplatina & Intravenosa & $\begin{array}{l}\text { Cancros do pâncreas, cabe- } \\
\text { ça/ pescoço, mama, gástrico, } \\
\text { pulmão e mesotelioma }\end{array}$ & III \\
\hline S-CKD602 & $\begin{array}{l}\text { Análogo da campto- } \\
\text { tecina }\end{array}$ & Intravenosa & Cancro do útero recorrente & $\mathrm{I} / \mathrm{II}$ \\
\hline OSI-211 & Lurtotecano & Intravenosa & $\begin{array}{l}\text { Cancros da cabeça/pescoço } \\
\text { e ovário }\end{array}$ & II \\
\hline INX-0125 & Vinorrelbina & Intravenosa & Tumores sólidos avançados & I \\
\hline INX-0076 & Topotecano & Intravenosa & Tumores sólidos avançados & I \\
\hline $\begin{array}{l}\text { Anamicina } \\
\text { lipossomal }\end{array}$ & Anamicina & Intravenosa & Leucemia linfoblástica aguda & $\mathrm{I} / \mathrm{II}$ \\
\hline
\end{tabular}

${ }^{a}$ Atualmente alguns fármacos ainda não têm nome comercial atribuído e por isso são designados por código. A informação apresentada na tabela foi compilada a partir da referência bibliográfica [53]. 


\subsubsection{Formulações lipossomais de combinações de fármacos anticancerígenos}

\subsection{Considerações gerais}

O uso de combinações de fármacos anticancerígenos tem sido a prática padrão no tratamento do cancro ao longo das últimas décadas. No entanto, a aplicação de lipossomas como nanotransportadores de combinações de fármacos anticancerígenos foi apenas descrita nos últimos anos [9, 13, 34].

Até ao momento não existem formulações lipossomais encapsulando uma combinação de fármacos na prática clínica (Tabela 17.4).

Como referido anteriormente na seção 17.3.1, as combinações de fármacos podem originar efeitos sinergísticos, aditivos ou antagonistas dependendo do rácio em que os fármacos são combinados [11]. Durante uma experiência in vitro, o rácio entre dois fármacos pode ser facilmente controlado. No entanto a translação do rácio considerado terapêutico in vitro para a prática clínica é muito difícil devido à farmacocinética, biodistribuição e metabolismo distintos assumidos pelos fármacos, na forma livre, após administração intravenosa [13, 55]. Sendo assim, a referida farmacocinética não controlada de cada fármaco resulta na exposição das células tumorais a concentrações de fármaco abaixo do nível terapêutico ou na exposição a um rácio antagonista que resulta numa perda de atividade terapêutica [6,9]. A incapacidade de controlar o rácio dos dois fármacos administrados durante a circulação sistémica e, principalmente, no tecido tumoral, pode explicar em parte a reduzida eficácia terapêutica de algumas combinações de fármacos em quimioterapia do cancro [6].

Os sistemas de entrega de fármacos, tais como os lipossomas, podem controlar a libertação dos dois fármacos encapsulados, mantendo um rácio específico de combinação durante a circulação sanguínea. Vários estudos demonstraram que este controlo rigoroso permite um aumento significativo de eficácia terapêutica comparando com os fármacos na forma livre administrados em combinação ou com formulações de cada fármaco individualmente $[6,9,13,54,55]$. 
Em 2006, Mayer et al. [13] foram os primeiros a investigar a importância de manter o rácio óptimo de combinação in vivo, mediante encapsulação em lipossomas. Estudos adicionais [9, 54] demonstraram que os efeitos resultantes da interação de dois fármacos in vitro podem ser transpostos para o cenário in vivo uma vez que os lipossomas podem sincronizar a farmacocinética e a biodistribuição dos fármacos encapsulados em combinação e, assim, permitir a entrega dos fármacos ao tecido tumoral no rácio pretendido (Figura 17.5, em baixo). Pelo contrário, após a injeção intravenosa de dois fármacos combinados na forma livre verifica-se que os fármacos se distribuem rapidamente para os tecidos saudáveis além do tumor, e o seu rácio torna-se diferente do rácio administrado (Figura 17.5, em cima) [6].

Sendo assim, é possível concluir que a nanotecnologia, por exemplo os lipossomas, constituem uma ferramenta valiosa para a avaliação pré-clínica de uma combinação de fármacos antes do desenvolvimento clínico. As vantagens da aplicação de uma combinação de fármacos lipossomais podem ser resumidas da seguinte forma [56]:

a) Injeção de múltiplos fármacos simultaneamente;

b) Perfil farmacocinético semelhante dos fármacos refletindo o perfil de farmacocinética do lipossoma transportador;

c) Controlo rigoroso da concentração dos fármacos no tecido tumoral alvo mediante controlo do rácio de combinação dos fármacos;

d) Métodos de preparação e composição lipídica das formulações lipossomais podem ser modelados para controlar a libertação dos fármacos;

e) Maior aceitação por parte do paciente e aumento da qualidade de vida pois o número de administrações e de efeitos adversos são reduzidos.

A empresa Celator Pharmaceuticals foi pioneira no desenvolvimento de formulações lipossomais de uma combinação de fármacos para tratamento do cancro. No presente momento, esta empresa possui algumas formulações em desenvolvimento clínico e pré-clínico, como indicado na Tabela $\mathbf{1 7 . 4}$. 

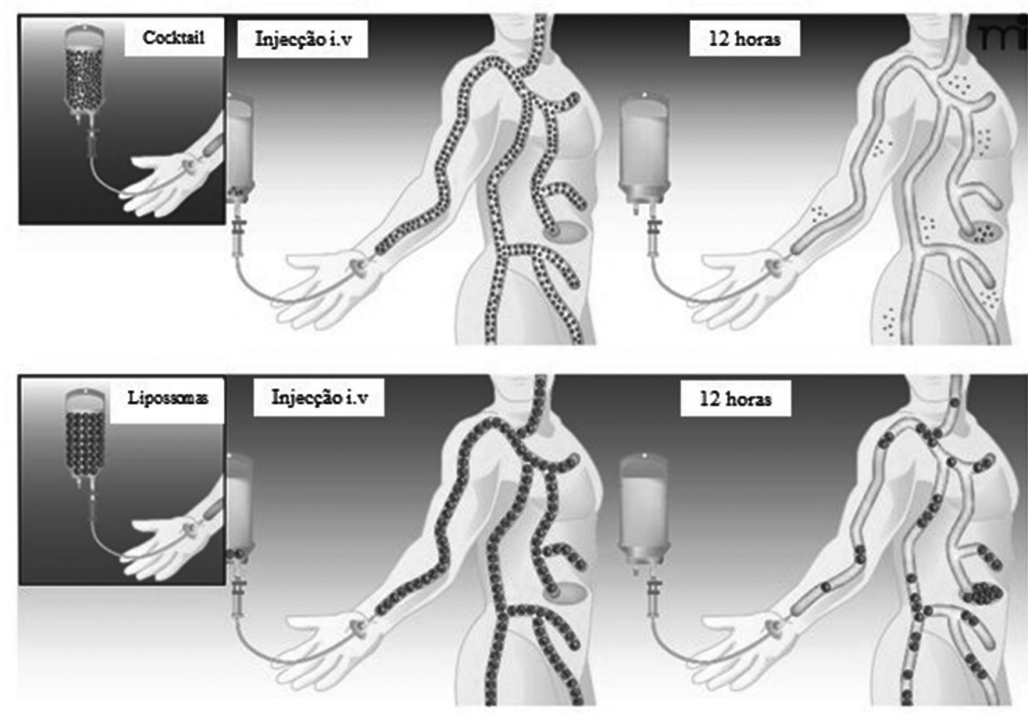

Figura 17.5. Descrição de como o sucesso da aplicação clínica de uma combinação de fármacos depende da manutenção in vivo e entrega tumoral dos fármacos no rácio de combinação pretendido. Em cima - após administração intravenosa de uma mistura de fármacos na forma livre, os fármacos distribuem-se independentemente e rapidamente pelos tecidos saudáveis e pelo tumor. Os fármacos alcançam o tumor num rácio de combinação diferente daquele que foi administrado. Em baixo - Os lipossomas mantêm na circulação sanguínea os fármacos encapsulados durante um longo período de tempo e entregam os mesmos ao tumor no rácio de combinação pretendido. Adaptado da referência bibliográfica [6].

Tabela 17.4. Formulações lipossomais de combinações de fármacos em desenvolvimento pela empresa Celator Pharmaceuticals (http://www.celatorpharma.com (2012)).

\begin{tabular}{c|l|l|l}
\hline Código & \multicolumn{1}{|c|}{ Combinação de fármacos lipossomal } & \multicolumn{1}{|c}{ Indicação terapêutica } & \multicolumn{1}{c}{ Fase } \\
\hline CPX-351 & Citarabina : daunorrubicina & Leucemia mieloide aguda & II \\
\hline CPX-1 & Hidroclorato de irinotecano:floxuridina & Cancro coloretal & II \\
\hline CPX-571 & Hidroclorato de irinotecano:cisplatina & $\begin{array}{l}\text { Cancro do pulmão de } \\
\text { pequenas células }\end{array}$ & Pré-clínico \\
\hline CPX-8XY & Desconhecido & Desconhecido & Investigação \\
\hline
\end{tabular}

\subsection{Desenvolvimento de formulações lipossomais para entrega de uma combinação de fármacos}

O conceito de combinar fármacos, com propriedades físico-químicas distintas, num único nanotransportador (ex. lipossoma) que encapsula 
eficazmente esses fármacos e os liberta in vivo com a mesma taxa, representa um grande desafio científico e técnico.

Presentemente, a encapsulação lipossomal de uma combinação de fármacos anticancerígenos representa um novo paradigma. Apesar desta abordagem nanotecnológica ser extremamente promissora, na literatura existe um número reduzido de artigos científicos publicados que reportam estudos bem-sucedidos de encapsulação eficaz de dois fármacos num único lipossoma [9, 13, 54, 57]. Esta limitação resulta das dificuldades técnicas associadas à obtenção de uma encapsulação eficiente e estável dos dois fármacos, assim como à manutenção e entrega tumoral do rácio fármaco 1: fármaco 2 após administração sistémica $[9,58]$.

Existem três diferentes estratégias para formular uma combinação de dois fármacos usando lipossomas:

a) Combinação de um fármaco lipossomal com um fármaco na forma livre

b) Encapsulação de cada fármaco num lipossoma individualmente e, posteriormente, combinação das duas formulações lipossomais

c) Combinação dos dois fármacos num único lipossoma mediante encapsulação simultânea ou sequencial.

As vantagens e limitações de cada estratégia são discutidas de seguida mantendo a ordem descrita:

a) Uma formulação lipossomal de um fármaco pode ser administrado simultaneamente com um fármaco livre mas interações lipossoma - fármaco livre podem ocorrer, tais como interações hidrofóbicas ou encapsulação do fármaco livre se o lipossoma apresentar um gradiente de pH [59-60]. Sendo assim, a ocorrência de interações desfavoráveis pode induzir alteração nos parâmetros farmacocinéticos dos fármacos livre e/ou encapsulado, podendo resultar numa redução da eficácia terapêutica e/ou aumento da toxicidade [59].

b) Talvez a abordagem mais simplista para coordenar a farmacocinética de uma combinação de fármacos seja encapsular cada fármaco individualmente num lipossoma e, posteriormente combinar 
numa única suspensão, as duas formulações lipossomais no rácio fármaco 1: fármaco 2 pretendido. No entanto, esta estratégia de desenvolver formulações lipossomais independentes e posteriormente administrá-las em conjunto a pacientes seria extremamente dispendiosa dados os custos elevados dos constituintes lipídicos e do processo de produção das duas formulações em separado [9].

c) A co-encapsulação de dois fármacos num único lipossoma é uma estratégia mais favorável em relação ao desenvolvimento de formulações lipossomais de fármacos individualmente uma vez que os custos de produção são reduzidos, a administração de carga lipídica ao paciente é minimizada (associada a efeitos adversos associados à infusão) e uma possível interferência de um lipossoma na farmacocinética do outro é eliminada $[9,58]$. Adicionalmente, uma co-encapsulação permite ultrapassar dúvidas quanto à biodistribuição dos fármacos que depende da composição lipídica do lipossoma que o transporta. Combinando dois fármacos num único lipossoma já não se irá verificar um metabolismo e eliminação independentes mas sim uma farmacocinética única induzida pelas características do veículo transportador (lipossoma). No entanto, esta estratégia representa um grande desafio técnico de forma a desenvolver uma formulação lipossomal que demonstre uma cinética de libertação semelhante para ambos os fármacos. Para tal, é necessário otimizar diversos parâmetros durante o desenvolvimento da formulação lipossomal (método de preparação, composição lipídica do lipossoma, rácio fármaco 1: lípido total, rácio fármaco 2: lípido total, rácio fármaco 1: fármaco 2 [9].

\subsection{Conclusões}

O desenvolvimento de uma combinação quimioterapêutica para tratamento do cancro deve ser baseada numa seleção racional de fármacos a combinar e numa avaliação in vitro sistemática e quantitativa dos efeitos de interação dependentes do rácio de combinação. Estudos in vitro 
que avaliam o efeito antiproliferativo de uma combinação de fármacos incubados com uma linha celular antitumoral mediante a utilização de um método quantitativo, permite uma preparação mais racional de um futuro protocolo quimioterapêutico na prática clínica.

A transposição de rácios de combinação específicos, previamente selecionados em estudos in vitro, para o cenário clínico é complexa devido à farmacocinética independente de cada fármaco presente na combinação após administração intravenosa. Esta farmacocinética não coordenada pode resultar na exposição das células tumorais a concentrações sub-terapêuticas ou a um rácio antagonista com concomitante perda de atividade terapêutica.

A extensa informação obtida nos estudos in vitro sobre a dependência dos efeitos antiproliferativo do rácio de combinação pode ser usada para formular combinações de fármacos em sistemas de entrega de fármaco nanotecnológicos. Os lipossomas são um excelente exemplo deste tipo de sistema uma vez que têm demonstrado capacidade de aumentar o índice terapêutico dos fármacos anticancerígenos mediante aumento da atividade antitumoral e/ou redução dos efeitos tóxicos nos tecidos saudáveis.

O sucesso clínico desta estratégia nanotecnológica no tratamento do cancro está dependente do desenvolvimento de uma formulação lipossomal, com propriedades específicas, que consiga encapsular os dois fármacos de uma forma eficiente e estável e, posteriormente, manter o rácio de combinação após administração intravenosa e expor o tumor ao rácio efetivo de combinação.

\subsection{Referências}

[1] R. Siegel, D. Naishadham, A. Jemal. CA Cancer. J. Clin. 2012, 62,10-29.

[2] D. Hanahan,R.A. Weinberg. Cell. 2011, 144, 646-74.

[3] J.T. Dong. J. Cell. Biochem. 2006, 97, 433-47.

[4] M. Suggitt, M.C. Bibby. Clin. Cancer Res. 2005, 11, 971-81.

[5] G.F. Ismael, D.D. Rosa, M.S. Mano,A. Awada. Cancer Treat. Rev. 2008, 34, 81-91.

[6] L.D. Mayer,A.S. Janoff. Mol. Interv. 2007, 7, 4, 216-23.

[7] E.C. Ramsay, N. Dos Santos, W.H. Dragowska, J.J. Laskin, M.B. Bally. Curr. Drug Deliv. 2005, 2, 341-51.

[8] W. Zoli, L. Ricotti, A. Tesei, F. Barzanti, D.Amadori. Crit. Rev. Oncol. Hematol. 2001, 37, 69-82. 
[9] T.O. Harasym, P.G. Tardi, N.L. Harasym, P. Harvie, S.A. Johnstone, L.D. Mayer. Oncol. Res. 2007, 16, 361-74.

[10] T.C. Chou. Cancer Res. 2010, 70, 440-6.

[11] T.C. Chou. Pharmacol Rev. 2006, 58, 621-81.

[12] J.H. Goldie. Cancer Metastasis Rev. 2001, 20, 63-8.

[13] L.D. Mayer, T.O. Harasym, P.G. Tardi, N.L. Harasym, C.R. Shew, S.A. Johnstone, E.C. Ramsay, M.B. Bally, A.S. Janoff. Mol. Cancer Ther. 2006, 5, 1854-63.

[14] D.R. Budman, A. Calabro,W. Kreis. Anticancer Drugs 2002, 13, 1011-6.

[15] J.L. Merlin. Anticancer Res. 1994, 14, 2315-9.

[16] T.C. Chou, P. Talalay. Adv. Enzyme Regul. 1984, 22, 27-55.

[17] T.C. Chou. Contrib. Gynecol. Obstet. 1994, 19, 91-107.

[18] T.C. Chou. J. Theor. Biol. 1976, 59, 253-76.

[19] T.C. Chou, R.J. Motzer, Y. Tong,G.J. Bosl. J. Natl. Cancer Inst. 1994, 86, 1517-24.

[20] W. Kreis, D.R. Budman, A. Calabro. Cancer Chemother Pharmacol. 2001, 47, 78-82.

[21] T.C. Chou. J. Lab. Clin. Med. 1998, 132, 6-8.

[22] T.M. Allen. Drugs 1998, 56, 747-56.

[23] T.L. Andresen, S.S. Jensen,K. Jorgensen. Prog. Lipid Res. 2005, 44, 68-97.

[24] L. Cattel, M. Ceruti,F. Dosio. Tumori 2003, 89, 237-49.

[25] H. Devalapally, A. Chakilam,M.M. Amiji. J. Pharm. Sci. 2007, 96, 2547-65.

[26] D. Peer, J.M. Karp, S. Hong, O.C. Farokhzad, R. Margalit, R. Langer. Nat. Nanotechnol. 2007, 2, 751-60.

[27] R.C. Dutta. Curr. Pharm. Des. 2007, 13, 761-9.

[28] B. Haley,E. Frenkel. Urol. Oncol. 2008, 26, 57-64.

[29] F. Alexis, J.W. Rhee, J.P. Richie, A.F. Radovic-Moreno, R. Langer, O.C. Farokhzad. Urol. Oncol. 2008, 26, 74-85.

[30] K. Cho, X. Wang, S. Nie, Z.G. Chen, D.M. Shin. Clin. Cancer Res. 2008, 14, 1310-6.

[31] T. Lammers, W.E. Hennink,G. Storm. Br J. Cancer 2008, 99, 392-7.

[32] A.D. Bangham, M.M. Standish,J.C. Watkins. J. Mol. Biol. 1965, 13, 238-52.

[33] M.L. Immordino, F. Dosio,L. Cattel. Int. J. Nanomedicine 2006, 1, 297-315.

[34] R.D. Hofheinz, S.U. Gnad-Vogt, U. Beyer,A. Hochhaus. Anticancer Drugs 2005, 16, 691-707.

[35] D.B. Fenske, A. Chonn,P.R. Cullis. Toxicol Pathol 2008, 36, 21-9.

[36] D.C. Drummond, O. Meyer, K. Hong, D.B. Kirpotin, D. Papahadjopoulos. Pharmacol. Rev. 1999, 51, 691-743.

[37] A. Gabizon, H. Shmeeda,Y. Barenholz. Clin. Pharmacokinet. 2003, 42, 419-36.

[38] T.M. Allen. Drugs 1997, 54 Suppl. 4, 8-14.

[39] T.M. Allen. Trends Pharmacol. Sci. 1994, 15, 215-20.

[40] D.D. Lasic, J.J. Vallner, P.K. Working. Curr. Opin. Mol. Ther. 1999, 1, 177-85.

[41] V.P. Torchilin. AAPS J. 2007, 9, E128-47.

[42] V.P. Torchilin. Nat. Rev. Drug Discov. 2005, 4, 145-60.

[43] G. Gregoriadis, A.T. Florence. Drugs. 1993, 45, 15-28. 
[44] A.A. Gabizon. Cancer Res. 1992, 52, 891-6.

[45] D.B. Fenske,P.R. Cullis. Methods Enzymol. 2005, 391, 7-40.

[46] A. Chonn, S.C. Semple,P.R. Cullis. J. Biol. Chem. 1992, 267, 18759-65.

[47] D. Papahadjopoulos, T.M. Allen, A. Gabizon, E. Mayhew, K. Matthay, S.K. Huang, K.D. Lee, M.C. Woodle, D.D. Lasic, C. Redemann, et al. Proc. Natl. Acad. Sci. U S A 1991, 88, 11460-4.

[48] A.A. Gabizon, H. Shmeeda,S. Zalipsky. J. Liposome. Res. 2006, 16, 175-83.

[49] H. Maeda, J. Wu, T. Sawa, Y. Matsumura, K. Hori. J. Control. Release. 2000, 65, 271-84.

[50] F. Yuan, M. Dellian, D. Fukumura, M. Leunig, D.A. Berk, V.P. Torchilin, R.K. Jain. Cancer Res. 1995, 55, 3752-6.

[51] S.A. Abraham, D.N. Waterhouse, L.D. Mayer, P.R. Cullis, T.D. Madden, M.B. Bally. Methods Enzymol. 2005, 391, 71-97.

[52] A. Gabizon, D. Goren, R. Cohen,Y. Barenholz. J. Control. Release. 1998, 53, 275-9.

[53] H.I. Chang,M.K. Yeh. Int. J. Nanomedicine. 2012, 7, 49-60.

[54] P. Tardi, S. Johnstone, N. Harasym, S. Xie, et al. Leuk. Res. 2009, 33, 129-39.

[55] R.J. Lee. Mol. Cancer Ther. 2006, 5, 1639-40.

[56] Y. Bae, T.A. Diezi, A. Zhao,G.S. Kwon. J. Control. Release. 2007, 122, 324-30.

[57] X. Zhao, J. Wu, N. Muthusamy, J.C. Byrd, R.J. Lee. J. Pharm. Sci. 2008, 97, 1508-18.

[58] P.G. Tardi, R.C. Gallagher, S. Johnstone, N. Harasym, M. Webb, M.B. Bally, L.D. Mayer. Biochim. Biophys. Acta. 2007, 1768, 678-87.

[59] D.N. Waterhouse, N. Dos Santos, L.D. Mayer,M.B. Bally. Pharm. Res. 2001, 18, 1331-5.

[60] L.D. Mayer, J. Reamer, M.B. Bally. J. Pharm. Sci. 1999, 88, 96-102. 